\title{
Crack initiation prediction of additive manufactured ductile nickel- based superalloys
}

\author{
Thomas Lindström ${ }^{1, *}$, Robert Eriksson ${ }^{1}$, Daniel Ewest ${ }^{1,2}$, Kjell Simonsson ${ }^{1}$, Jan-Erik Lundgren ${ }^{2}$, and Daniel Leidermark ${ }^{1}$ \\ ${ }^{1}$ Division of Solid Mechanics, Linköping University, SE-58183 Linköping, Sweden \\ ${ }^{2}$ Siemens Industrial Turbomachinery AB, SE-61283 Finspång, Sweden
}

\begin{abstract}
A model to predict crack initiation life of an additive manufactured nickel-based superalloy similar to Hastelloy $X$ subjected to low-cycle fatigue loading at room temperature has been developed, taking material anisotropy into account. An anisotropic constitutive model based on the Hill yield criterion was developed, with linear kinematic hardening up to a saturation value of the back stress, above which the material behaves perfectly plastic. Low-cycle fatigue experiments has been performed on additive manufactured smooth bars with two different build orientations, with an angle of $0^{\circ}$ and $90^{\circ}$ relative to the building platform. A total of 20 experiments at room temperature were conducted with different strain ranges and $R$-values. To predict the crack initiation life of the specimens, a model based on the Smith-Watson-Topper (SWT) parameter has been established, where ten of the specimens were used to calibrate the initiation model, and the remaining specimens were used for validation. Using this model, the obtained crack initiation life agrees well with the experiments.
\end{abstract}

\section{Introduction}

Due to the increasing use of renewable energy on the energy market, usage of stationary gas turbines has changed and their role have more become to stabilize the power grid. As a consequence, the running profile of gas turbines will be more cycle-driven, and the components will be exposed to a more fatigue related loading which must be taken into consideration during the design [1]. In order to optimize gas turbine components with respect to efficiency, fuel flexibility and performance, additive manufacturing (AM) has been identified as a major technology due to the possibility to create more complex geometries, and to simplify assemblies of previously multiple components based on conventional manufacturing techniques.

Selective laser melting (SLM) is an AM technique where a thin layer of metallic powder is deposited on a substrate, on which a laser is melting the powder, creating one layer of solid material. A new layer of powder is then spread out and the procedure continues until a complete component is achieved. In SLM, the material will receive a small scale weld-like microstructure. The build platform is held at constant temperature during the process, causing rapid solidification of the material and high temperature gradients. These gradients affect the microstructure by generating elongated grains in the building direction which is the source of the anisotropic behaviour of the material. This has been observed in numerous studies for different alloys, c.f. [2-7]. Components for combustor applications in gas turbines is one example where AM has already been successfully adopted [8]. The use of
AM can improve the performance of such components with respect to fuels of different nature and the manufacturing technique can be used to simplify repair and decrease costs. Combustor components in a gas turbine are exposed to high temperatures and large mechanical loads. To withstand the loading conditions, materials with satisfying properties for this working environment have to be used. Combustor components are therefore advantageously manufactured by ductile nickel-based superalloys, due to their oxidation resistance and strength at high temperature.

In order to fully understand how the manufacturing process affects the life of SLM ductile nickel-based superalloy components, accurate prediction models for crack initiation and propagation must be developed, taking material anisotropy into account. Due to the complex loadings of combustor components with cyclic thermal and mechanical loads, SLM manufactured components exposed to thermomechanical fatigue must be analysed and evaluated. In addition, for thermomechanical fatigue loads, the room temperature behaviour is of great importance, since crack growth can be present during e.g. an out-of-phase loading condition. The aim of this paper is to evaluate the crack initiation behaviour and life of SLM manufactured smooth bars exposed to low-cycle fatigue (LCF) loadings at room temperature. The material used is an alloy similar to Hastelloy X, i.e. a ductile nickel-based superalloy with superior oxidation and high temperature strength. A total of 20 tests have been performed with different strain ranges and $R$-values using specimens manufactured in $0^{\circ}$ and $90^{\circ}$ direction relative to the building platform. A material model, that takes material anisotropy into

*Corresponding author: thomas.lindstrom@liu.se 
account, has been developed to describe the material behaviour. Furthermore, a relation based on the SmithWatson-Topper (SWT) parameter has been adopted to predict the initiation life of the specimens, where ten of the specimens have been used to calibrate the model, while the remaining ten have been used for validation.

\section{Experiments}

As mentioned previously, the investigated material used in the LCF experiment was a ductile nickel-based superalloy similar to Hastelloy X. To capture the anisotropic behaviour due to manufacturing, the specimens were built in two different orientations relative the building platform; $0^{\circ}$ and $90^{\circ}$, as illustrated in Fig. 1.

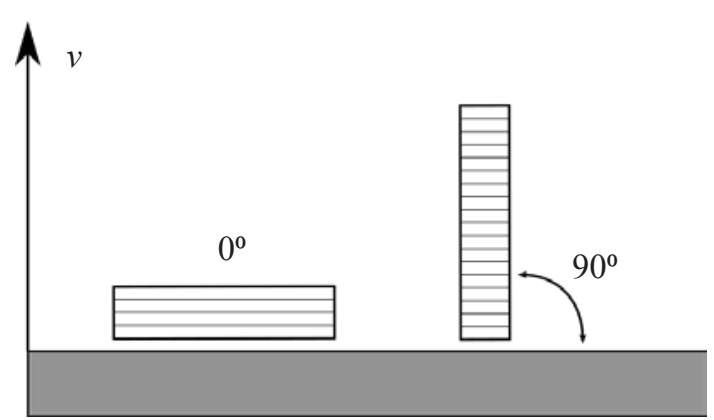

Fig. 1: Illustration of how the test specimens were manufactured, where $v$ is the building direction. $0^{0}$ specimen (left) manufactured with an angle of $0^{\circ}$ to the build platform and $90^{\circ}$ (right) manufactured with an angle of $90^{\circ}$ from the build platform.

A total of 20 strain controlled LCF experiments were performed with different strain ranges for two $R$-values and building orientations; $R=0$ and -1 , and $0^{\circ}$ and $90^{\circ}$, respectively. The data used in the experiments can be seen in Table 1. The crack initiation lives were taken as $5 \%$ load-drop from the trend line of the maximum stress in each cycle.

The specimens were manufactured as cylindrical smooth bars with optimized process parameters in an EOS M290 machine with building layers of $40 \mu \mathrm{m}$. As usual when manufacturing SLM components, the scan direction of the laser was rotated in the building plane after every built layer. Due to this rotation, the material properties in the plane parallel to the building platform becomes more or less uniform. The material is therefore assumed to be transversely isotropic, since the material properties in the building direction is different due to the elongated grain structure.

To avoid effects from the surface during fatigue testing, the as-machined diameter of the specimens were $16 \mathrm{~mm}$, and then machined to an hourglass geometry by conventional techniques to the final diameter of $6.35 \mathrm{~mm}$ in the gauge section.

The gauge length of the extensometer for the experiments was $11.4 \mathrm{~mm}$.
Table 1 : Data used in the experiments for all specimens.

\begin{tabular}{|c|c|c|c|}
\hline & Specimen & & $\Delta \varepsilon[\%]$ \\
\hline \multirow[t]{10}{*}{$0^{0}$} & S0:1 & \multirow[t]{5}{*}{$\mathrm{R}=0$} & 0.7 \\
\hline & S0:2 & & 0.9 \\
\hline & S0:3 & & 1.3 \\
\hline & S0:4 & & 1.8 \\
\hline & S0:5 & & 2.5 \\
\hline & S0:6 & \multirow[t]{5}{*}{$R=-1$} & 0.7 \\
\hline & S0:7 & & 0.9 \\
\hline & S0:8 & & 1.1 \\
\hline & S0:9 & & 1.5 \\
\hline & S0:10 & & 2.0 \\
\hline \multirow[t]{10}{*}{$90^{\circ}$} & S90:1 & \multirow[t]{5}{*}{$\mathrm{R}=0$} & 0.6 \\
\hline & S90:2 & & 0.8 \\
\hline & S90:3 & & 1.0 \\
\hline & S90:4 & & 1.3 \\
\hline & S90:5 & & 2.6 \\
\hline & S90:6 & \multirow[t]{5}{*}{$\mathrm{R}=-1$} & 0.8 \\
\hline & S90:7 & & 1.0 \\
\hline & S90:8 & & 1.5 \\
\hline & S90:9 & & 1.99 \\
\hline & S90:10 & & 3.0 \\
\hline
\end{tabular}

In Fig. 2, the applied strain range is plotted against the crack initiation lives obtained from the experiments for the $0^{\circ}$ and the $90^{\circ}$ specimens, where the crack initiation lives are normalized with the longest life obtained from experiments. As can be seen, the material shows anisotropic behaviour for LCF loading in room temperature, where the anisotropic crack initiation behaviour is most evident for large strain ranges.

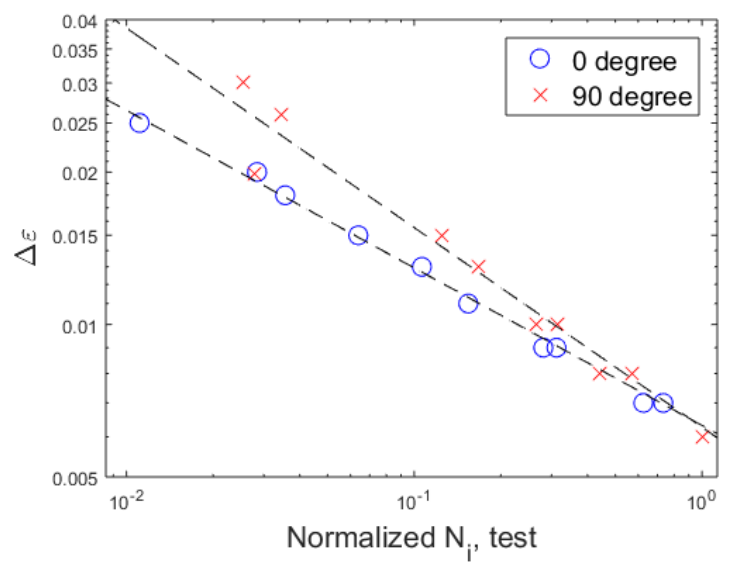

Fig. 2 : Nominal strain range, $\Delta \boldsymbol{\varepsilon}$, plotted against normalized cycles to crack initiation, $\boldsymbol{N}_{\boldsymbol{i}}$, obtained from experiments.

As can be seen from Fig. 2, there is a difference between the two building orientations. One can also see that the $90^{\circ}$ specimens have longer initiation life compared to $0^{\circ}$ specimens, which agrees with several other studies on SLM nickel-based superalloy, see e.g. [2].

When studying the fracture surface of the specimens, the anisotropic behaviour is also evident. In Fig. 3, two specimens with the same loading condition but different building orientations are shown. Comparing the $0^{0}$ 


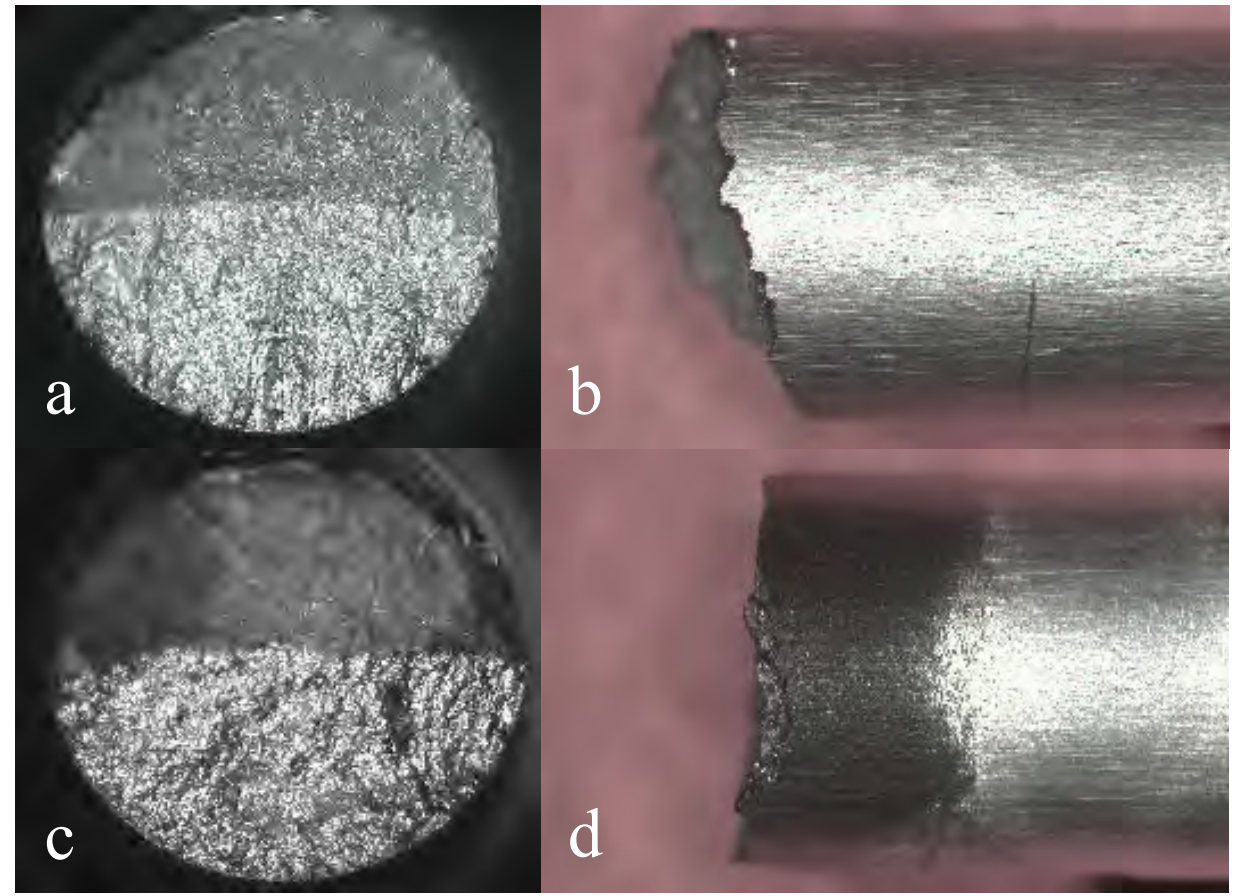

Fig. 3: Fracture appearance for specimen $\mathrm{S} 0: 3$ a) shown from above, b) from the side, and for specimen S90:4 c) from above and d) from the side.

specimens with the $90^{\circ}$ specimens, one can see that the former specimens have a much smoother fracture surface compared to the latter. Furthermore, by looking at the specimens from the side, the fracture of the $90^{\circ}$ specimens seem to appear on a plane orthogonal to the loading direction, while the fracture plane for the $0^{0}$ specimens are tilted w.r.t. the orthogonal plane to the loading.

\section{Modelling}

\subsection{Constitutive modelling}

In order to describe the crack initiation behaviour of the material, a transversely isotropic material model was developed, catching the elasto-plastic anisotropic behaviour in the context of small deformations. In the elastic region the stresses in a component subjected to applied strains, can be calculated as:

$$
\sigma_{i j}=C_{i j k l}^{e} \varepsilon_{k l}^{e}
$$

where $C_{i j k l}^{e}$ is the fourth-order elastic stiffness tensor. Due to transversely isotropic material behaviour, the configuration of the elastic stiffness tensor is dependent on the material direction in the global coordinate system. The material direction is specified by the normal unit vector to the material plane in which the material properties are isotropic. In the later described FE model, the cylindrical bar is modelled with the axial direction in the $z$-direction in the global coordinate system. Therefore, according to Fig. 1, a $90^{\circ}$ specimens corresponds to a material direction $\boldsymbol{v}=\left[\begin{array}{lll}0 & 0 & 1\end{array}\right]^{T}$ and a $0^{0}$ specimen corresponds to any unit vector in the $x-y$ plane, here adopted as $\boldsymbol{v}=\left[\begin{array}{lll}0 & 1 & 0\end{array}\right]^{T}$.
Since the constitutive relations for the material are dependent on the material direction, structural tensors have been used to describe the anisotropic material behaviour. For a transversely isotropic material, the structural tensor is calculated based on the material direction [9] as:

$$
M_{i j}=v_{i} v_{j}
$$

In the case of transversely isotropic materials, the elastic behaviour can be described with five independent elastic material parameters. Two of these, $\lambda$ and $\mu_{T T}$, describe the isotropic behaviour, while the three other constants, $\mu_{L T}, \alpha$ and $\beta$, are used to describe the anisotropic behaviour. From this, the elastic stiffness tensor is calculated as:

$$
\begin{aligned}
& C_{i j k l}^{e}=\lambda\left(I_{i j} I_{k l}\right)+\mu_{T T}\left(I_{i k} I_{j l}+I_{i l} I_{j k}\right) \\
& \quad+\alpha\left(M_{i j} I_{k l}+I_{i j} M_{k l}\right)+\beta\left(M_{i j} M_{k l}\right)+ \\
& \left(\mu_{L T}-\mu_{T T}\right)\left(I_{i k} M_{j l}+M_{i k} I_{j l}+I_{i l} M_{j k}+M_{i l} I_{j k}\right)
\end{aligned}
$$

where $I_{i j}$ is the second order unit tensor. For a $90^{\circ}$ specimen, i.e. $\boldsymbol{v}=\left[\begin{array}{lll}0 & 0 & 1\end{array}\right]^{T}$, the elastic stiffness tensor in Voigt notation becomes:

$\boldsymbol{C}^{e}=\left[\begin{array}{cccccc}\lambda+2 \mu_{T T} & \lambda & \lambda+\alpha & 0 & 0 & 0 \\ \lambda & \lambda+2 \mu_{T T} & \lambda+\alpha & 0 & 0 & 0 \\ \lambda+\alpha & \lambda+\alpha & \lambda+2 \alpha+\beta & 0 & 0 & 0 \\ 0 & 0 & 0 & \mu_{T T} & 0 & 0 \\ 0 & 0 & 0 & 0 & \mu_{L T} & 0 \\ 0 & 0 & 0 & 0 & 0 & \mu_{L T}\end{array}\right]$ 
For elasto-plastic materials undergoing small deformations, the total mechanical strain can be decomposed into one elastic and one plastic part:

$$
\varepsilon_{i j}=\varepsilon_{i j}^{e}+\varepsilon_{i j}^{p}
$$

The elastic part of the strain tensor follows the relation described in Eq. (1), and the plastic part follows the associated flow rule as:

$$
\mathrm{d} \varepsilon_{i j}^{p}=\mathrm{d} \lambda \frac{\partial f}{\partial \sigma_{i j}}
$$

where $\mathrm{d} \lambda$ is the plastic multiplier and $\partial f / \partial \sigma_{i j}$ the direction of the plastic flow, where $f$ describes the yield surface. Considering the anisotropic yield behaviour of the material, a Hill yield criterion is used where the parameters describe the different yield limits for the material in the different directions [10].

The yield function is expressed, in similarity with [1], as

$$
f=\sqrt{\sigma_{y}^{2}\left(s_{i j}-\alpha_{i j}\right) P_{i j k l}\left(s_{k l}-\alpha_{k l}\right)}-\sigma_{y}=0
$$

where $s_{i j}$ is the deviatoric stress tensor, $\alpha_{i j}$ is the back stress tensor and $P_{i j k l}$ is the fourth order anisotropic tensor describing the directional dependent yield behaviour of the material. The anisotropic tensor contains the Hill parameters, and is dependent on the material direction. Therefore, this anisotropic tensor is also built up with use of the structural tensor as:

$$
\begin{gathered}
P_{i j k l}=(F+7 G+4 N-8 L)\left(M_{i j} M_{k l}\right) \\
+(3 G-N)\left(M_{i j} I_{k l}+I_{i j} M_{k l}\right) \\
+2(L-N)\left(M_{i k} I_{j l}+I_{i k} M_{j l}\right)+N\left(2 I_{i k} I_{j l}-I_{i l} I_{j k}\right) \\
-G\left(I_{i j} I_{k l}\right)+(N+2 L)\left(M_{i l} I_{j k}+I_{i l} M_{j k}\right)
\end{gathered}
$$

where $N=F+2 G$. For a $90^{\circ}$ specimen, the anisotropic tensor in Voigt notation becomes:

$$
\boldsymbol{P}=\left[\begin{array}{cccccc}
F+G & -G & -F & 0 & 0 & 0 \\
-G & F+G & -F & 0 & 0 & 0 \\
-F & -F & 2 F & 0 & 0 & 0 \\
0 & 0 & 0 & 2(F+2 G) & 0 & 0 \\
0 & 0 & 0 & 0 & 2 L & 0 \\
0 & 0 & 0 & 0 & 0 & 2 L
\end{array}\right]
$$

where $F, G$ and $L$ are the Hill parameters, defined as:

$$
\begin{gathered}
F=\frac{1}{2\left(\sigma_{L T, Y}\right)^{2}} \\
G=\frac{1}{\left(\sigma_{T T, Y}\right)^{2}}-F
\end{gathered}
$$

$$
L=\frac{1}{2\left(\tau_{Y}\right)^{2}}
$$

where $\sigma_{L T, Y}$ is the yield stress in the direction orthogonal to the building platform, $\sigma_{T T, Y}$ is the yield stress in the direction parallel to the building direction, and $\tau_{Y}$ is the anisotropic shear yield stress, which is not of importance when only considering homogeneous uniaxial loading of $0^{\circ}$ and $90^{\circ}$ specimens.

In Eq. (7), the constant scalar $\sigma_{y}$ is defined as:

$$
\sigma_{y}=\left(\frac{3}{2(2 F+G)}\right)^{1 / 2}
$$

Note that, for $F=G$ and $L=3 G$, one ends up with the traditional von Mises yield criterion.

Furthermore, the direction of the plastic flow becomes:

$$
\frac{\partial f}{\partial \sigma_{i j}}=\frac{\sigma_{y}^{2} P_{i j k l}\left(s_{k l}-\alpha_{k l}\right)}{\sqrt{\sigma_{y}^{2}\left(s_{m n}-\alpha_{m n}\right) P_{m n p q}\left(s_{p q}-\alpha_{p q}\right)}}
$$

The material is assumed to follow a linear kinematic hardening behaviour, with a saturation value above which the material acts as a perfect plastic material. To capture the saturation of the back stress, the back stress is multiplied with a scalar function, $H$, which quenches the evolution of it at a certain saturation value.

$$
\mathrm{d} \alpha_{i j}=\frac{2}{3} C^{p} \mathrm{~d} \varepsilon_{i j}^{p} H\left(g_{s a t}\right)
$$

where $C^{p}$ is the linear kinematic hardening coefficient, which is dependent on the material orientation. To take this into account, for a specimen with the axial direction and the applied load in the $z$-direction in the global coordinate system, $C^{p}$ is calculated as:

$$
C^{p}=M_{i j} A_{i j k l} M_{k l}
$$

The fourth order tensor $A_{i j k l}$ contains the kinematic hardening coefficients in each direction. When only considering $0^{\circ}$ and $90^{\circ}$ specimens, $A_{i j k l}$ can, in Voigt notation, be written:

$$
\boldsymbol{A}=\left[\begin{array}{cccccc}
C_{T T}^{p} & 0 & 0 & 0 & 0 & 0 \\
0 & C_{T T}^{p} & 0 & 0 & 0 & 0 \\
0 & 0 & C_{L T}^{p} & 0 & 0 & 0 \\
0 & 0 & 0 & 0 & 0 & 0 \\
0 & 0 & 0 & 0 & 0 & 0 \\
0 & 0 & 0 & 0 & 0 & 0
\end{array}\right]
$$

where $C_{T T}^{p}$ and $C_{L T}^{p}$ are the linear kinematic hardening coefficient in the direction parallel and orthogonal to the building platform, respectively.

In Eq. (16), the structural tensor is used to pick out the kinematic hardening coefficient in the current direction. In addition, in Eq. (15), $\left.\mathrm{H}^{*}\right)$ is the Heaviside function and the expression inside the Heaviside, $g_{\text {sat }}$, accounts 
for the saturation of the back stress tensor in the following way:

$$
g_{s a t}=\alpha_{s a t}-\frac{\partial f}{\partial \sigma_{i j}} \alpha_{i j}
$$

where $\alpha_{\text {sat }}$ is the magnitude of the saturation value of the back stress, which has been found to be linearly proportional to the kinematic hardening coefficient for this material. The saturation value of the back stress can therefore be calculated by scaling the kinematic hardening coefficient by the scalar $w$, according to:

$$
\alpha_{\text {sat }}=w^{p} C^{p}
$$

This constitutive model has similar features as the OhnoWang Model 1, further described in [11], but takes anisotropic material behaviour into account through the anisotropic tensor $P_{i j k l}$. Furthermore, the definition of the saturation value of the back stress differs between the above described model and the Ohno-Wang Model 1.

The material parameters have been extracted from stabilized hysteresis loops at mid-life of the specimens, and the parameters were taken as the mean of the values from the experiments for the two different build orientations.

\subsection{Crack initiation model}

From the experiments, it was observed that using the SWT parameter [12] to describe the initiation life, one can capture the differences between the two building orientation and establish a relation for the crack initiation life:

$$
\sigma^{\max } \varepsilon^{\mathrm{a}}=a\left(N_{i}\right)^{b}
$$

where the left-hand side of the expression is the SWT parameter, $\sigma^{\max }$ is the maximum stress, $\varepsilon^{\mathrm{a}}$ is the strain amplitude, $N_{i}$ is the number of cycles to crack initiation and $a, b$ are constants determined by calibration. The maximum stress and the strain amplitude is extracted at mid-life of the specimens. Fig. 4 shows the SWT parameter, normalized with the largest SWT parameter calculated from the experiments, against normalized cycles to crack initiation observed from LCF experiments.

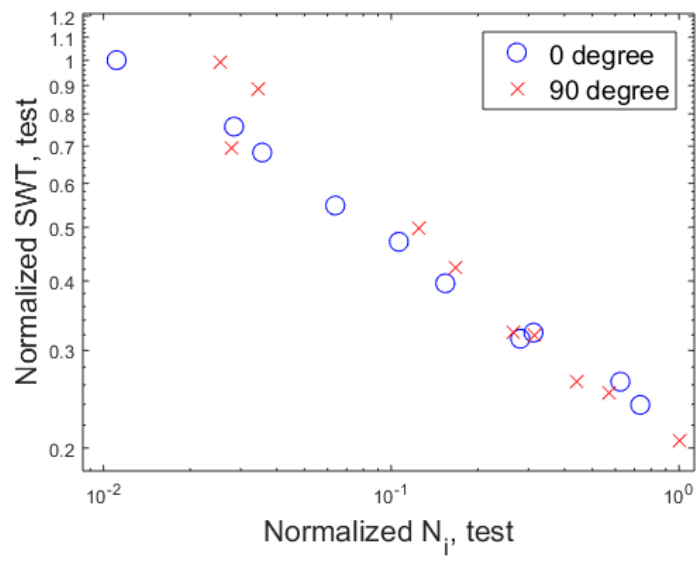

Fig. 4: Normalized SWT parameter plotted against normalized cycles to crack initiation, $\boldsymbol{N}_{\boldsymbol{i}}$, from the experiments.

\section{Calibration and evaluation}

Simulations of the LCF experiments were performed using the finite element program ABAQUS 6.12 [13], with the above described material description implemented as a user defined material model. Only the part of the specimen inside the extensometer region has been modelled. A total of 320 8-noded linear brick elements with reduced integration was used. The load was applied as a cyclic displacement in the axial direction on the top on the specimen and the bottom surface was fixed in the axial direction. The remaining boundary conditions were set so that the cross section of the specimen was allowed to contract. Since crack initiation is assumed to occur at the surface of the specimen, the stresses and strains were extracted from an element on the surface of the specimen. Furthermore, to avoid undesirable impact from the boundary conditions on the extracted result, the chosen element was picked as far away from the boundary as possible.

In the FE simulations, one loading cycle, with start and finish in tension has been simulated. The maximum stress was then extracted at the end of the load cycle.

In Fig. 5, a simulated hysteresis loop of specimen S0:5, c.f. Table 1, is shown. Comparing the maximum and minimum stresses for this simulated hysteresis with the values obtained from the experiment, it can be seen that the material model predicts these values with very good accuracy. 


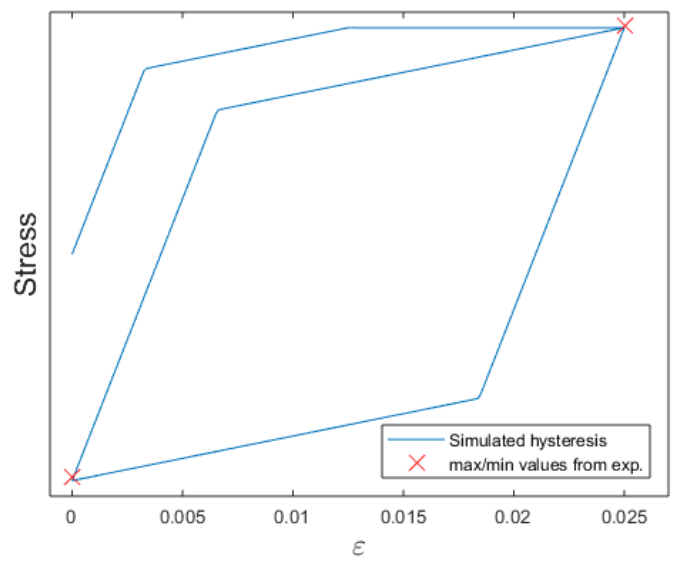

Fig. 5: Simulated hysteresis loop for specimen S0:5 compared to obtained maximum and minimum stress at mid-life from the experiment.

The $0^{0}$ specimens (specimen S0:1-10 in Table 1) were used to calibrate the model. The SWT parameter of these specimens was extracted from the FE model and plotted against the crack initiation lives obtained from the experiments in a log-log-scale. The constants $a$ and $b$ from the crack initiation model, Eq. (20), were then evaluated by a curve fit of the SWT parameter and the crack initiation lives. In Fig. 6, the normalized crack initiation lives from the FE simulations of the $0^{0}$ specimens are plotted against the normalized crack initiation lives obtained from the experiments. The scatter bands shows the standard deviation of the simulation results compared to experimental data.

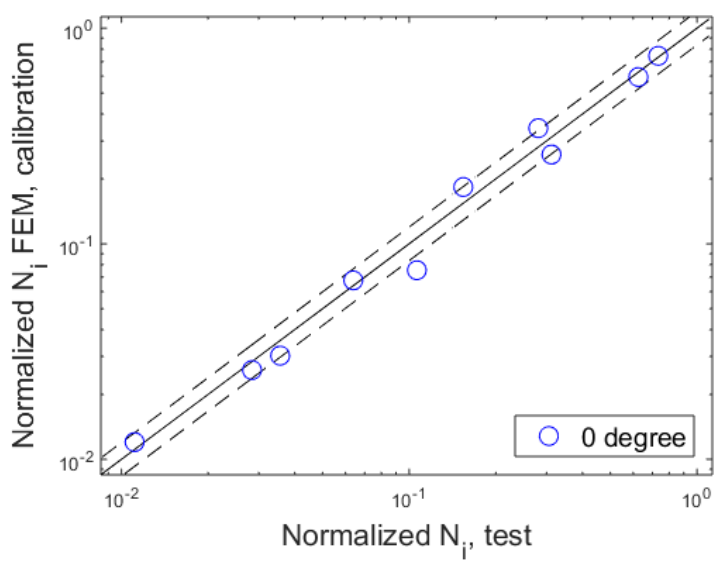

Fig. 6 : Normalized crack initiation lives, $\boldsymbol{N}_{\boldsymbol{i}}$, evaluated from the simulations for the calibrated specimens plotted against the normalized crack initiation lives from the experiments.

As a validation, the calibrated model was then used to predict the crack initiation lives of the $90^{\circ}$ specimens (specimen S90:1-10 in Table 1). The results of this is shown in Fig. 7, where the predicted normalized crack initiation lives are plotted against the normalized lives obtained in the experiments. The scatter bands plotted in Fig. 7 are the standard deviation of the $0^{0}$ specimens compared to the experiments, multiplied with a factor of 2.3.

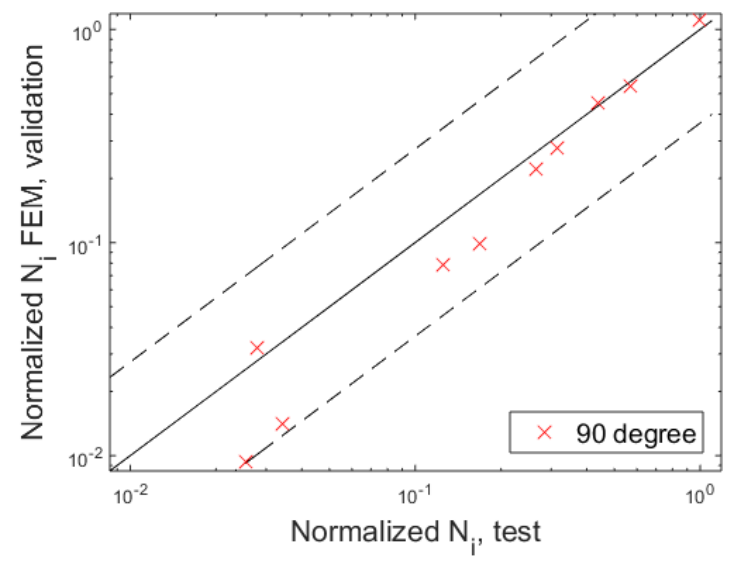

Fig. 7 : Normalized crack initiation lives, $\boldsymbol{N}_{\boldsymbol{i}}$, evaluated from simulations for the validated specimens plotted against the normalized crack initiation lives from the experiments.

The SWT parameter calculated with the FE model and the calculated SWT parameter extracted from the experiments are plotted against each other in Fig. 8. The largest deviation of the simulated SWT parameter compared to the SWT parameter calculated from experimental data is $10 \%$, which indicates that the developed material model predicts the maximum stress during a mid-life load cycle with good agreement to experiments.

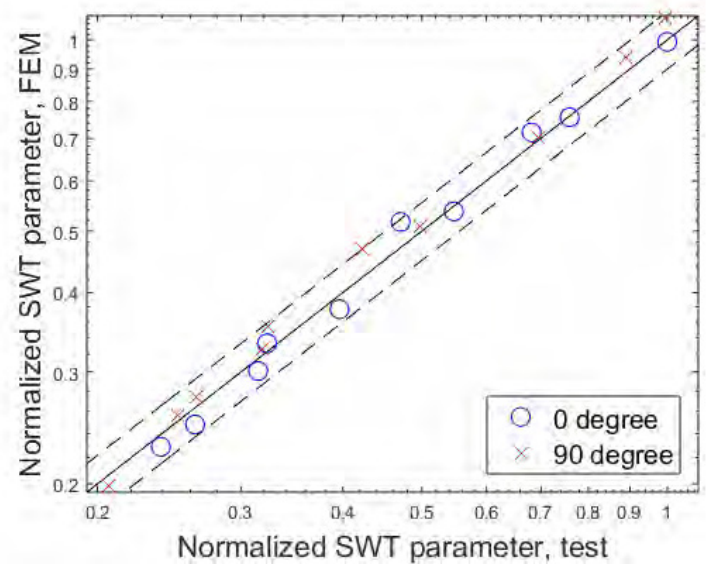

Fig. 8: Comparison of the normalized SWT parameter extracted from FE analyses and those calculated from experiments.

\section{Discussion}

The developed initiation model enables the prediction of the initiation life of both $0^{\circ}$ and $90^{\circ}$ specimens with the same relation with reasonable agreement to experiments. The crack initiation lives of all specimens used for validation are within 2.3 standard deviations of the specimens used for calibration, and the specimens with the largest applied strain ranges deviates most from the experimentally obtain lives. This indicates that there might be some further mechanism affecting the initiation life. Another thing that could affect the results is that all the material properties are taken as a mean value from experimental observations. An example of this is shown in Fig. 9, where the experimental elastic stiffness at mid- 
life of each specimen is plotted against the applied strain range. As can be seen, due to damage of the material, the elastic stiffness at mid-life decreases with increasing strain range.

In the presented crack initiation model, all the data are taken from mid-life of the component where a stable hysteresis is assumed. However, earlier loading cycles in the life of the components could possibly also affect the crack initiation life.

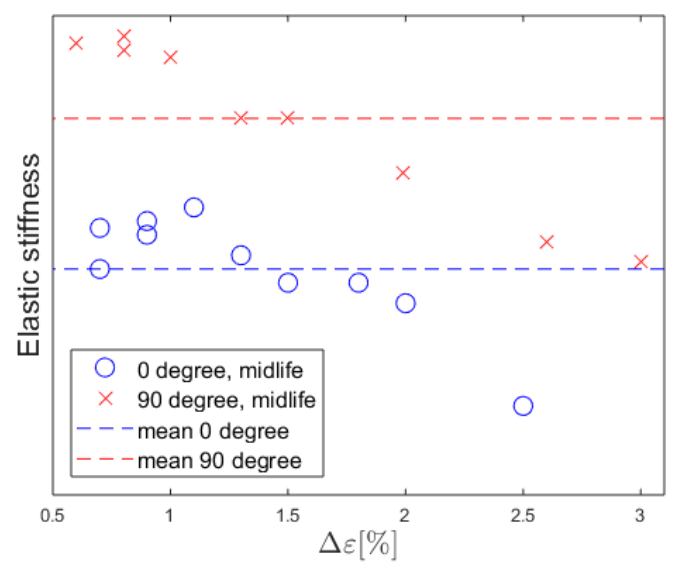

Fig. 9: Elastic stiffness plotted against the total strain range of the specimens at mid-life.

With the use of structural tensors to establish the constitutive relations for the material, an easy and straight forward approach to define the material orientation is obtained. The only input is the unit normal vector to the building platform on which the component is manufactured. The established material model can be used for any material orientation, not only those studied in this paper. This simplifies future studies on the material, when other material orientations might be in focus.

The developed material model has, similar to the OhnoWang Model 1, a linear kinematic hardening behaviour up to a saturation value of the back stress above which the material acts as a perfect plastic material. This material model predicts the maximum and minimum stress at a mid-life cycle with good agreement compared to experiments. Therefore, this model also predicts the crack initiation life accurately, since the only parameter needed from the FE simulations is the maximum stress when analyzing strain controlled smooth bars. Hence, it is not necessary in this case to use the complete hysteresis loop. However, when notched specimens are studied, a full hysteresis loop need to be considered, since local stresses and strains differ from global entities. This study has been performed under the assumption that only the building orientation affects the material properties. However, other research, e.g. [14], have found that also the amount of material surrounding the melt pool during manufacturing can influence the material properties. This has not been taken into account in this work, but is worth having in mind for future studies.

From the fractography, a clear difference can be seen between the $0^{\circ}$ specimens and the $90^{\circ}$ specimens. The fracture surface of the $0^{\circ}$ specimens is much smoother than on the $90^{\circ}$ specimens, which could be due to the elongated grains in the loading direction for the $90^{\circ}$ specimens. Furthermore, when studying the fracture surfaces from the side of the specimens, see Fig. $3 b$, the fracture of the $0^{0}$ specimens does not seem to appear at the plane orthogonal to the loading, which could be explained by the anisotropic material properties in the plane orthogonal to the loading.

From Fig. 2 it can be observed that one of the $90^{\circ}$ specimens with a strain range of $1.99 \%$ (specimen S90:9) collapses with the trend line of the $0^{\circ}$ specimens and experiences almost similar life as the $90^{\circ} 3 \%$ strain range specimen (specimen S90:10). Comparing the fracture surface of specimen S90:9 (Fig. 10b) with the one for specimen S90:8 (Fig. 10a), which have an applied strain range of $1.5 \%$, it is not clear if there is a different governing fracture mechanisms between these. However, one can see a slight difference close to the specimen surface of the where specimen S90:9 have a more irregular fracture surface than specimen S90:8. Studying the fracture surface of specimen S90:10 (Fig. $10 \mathrm{c}$ ), one can see a clear distinction compared to the other two specimens, indicating that at such large strain ranges, there is another governing fracture mechanism. 


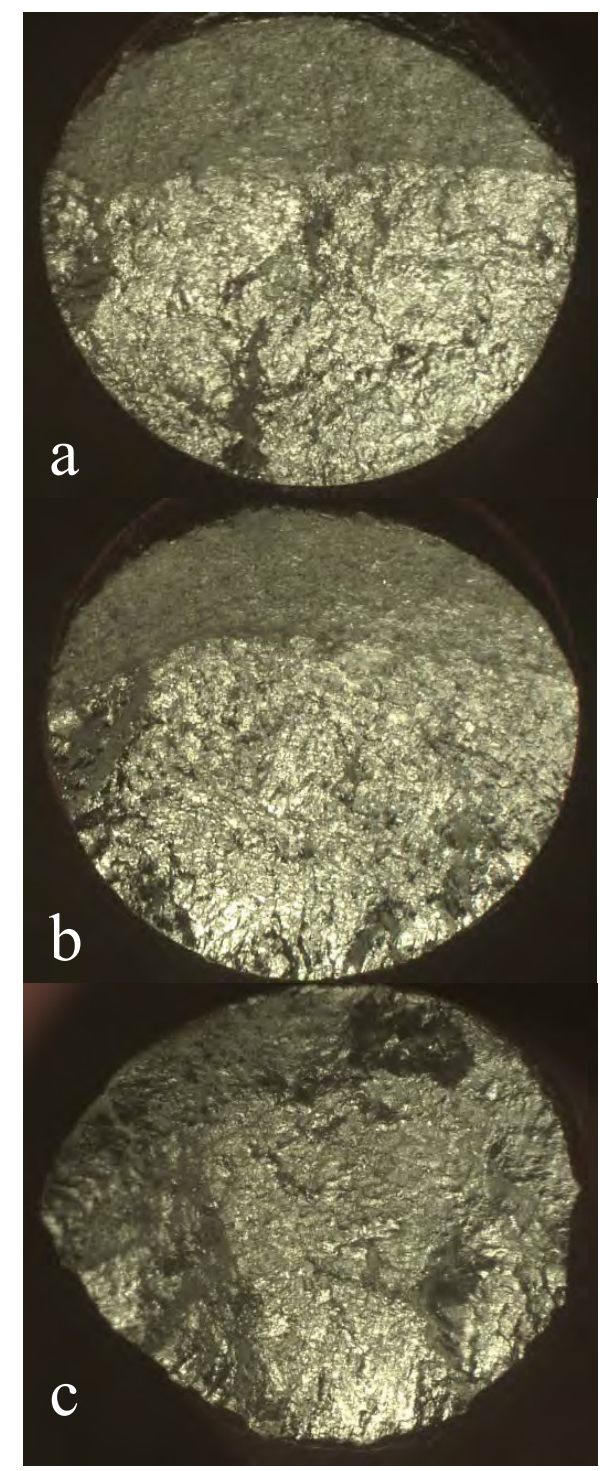

Fig. 10: Fracture surfaces of specimen a) S90:8 b) S90:9 and c) $S 90: 10$.

\section{Conclusions}

In this paper, a model for predicting crack initiation life for an SLM nickel-based superalloy subjected to LCF loading at room temperature has been established. The following conclusions can be drawn:

- A simple elasto-plastic constitutive model, with similar performance as the Ohno-Wang Model 1, that takes material anisotropy into account through structural tensor has been developed. This model can be used to simulate the elasto-plastic behaviour of the studied material with good agreement.

- The use of structural tensors for defining the transversely isotropic behaviour simplifies the modelling framework, as the building direction is a known entity from the manufacturing process.

- The adopted SWT model predicts the crack initiation lives in the two different building orientations and $R$ - values with good correlation to the performed experiments.

- The studied material clearly exhibit anisotropic crack initiation behaviour for LCF loading at room temperature, which has been confirmed through both experiments and by fractography.
This research has been funded by the Swedish Energy Agency and Siemens Industrial Turbomachinery AB through "Turbines for Future Energy Systems" (Turbiner för framtidens energisystem), Grant No. 44112-1, the support of which is gratefully acknowledged. The authors would also like to thank Linnéa Tingö at Siemens Industrial Turbomachinery $\mathrm{AB}$ for the laboratory work.

\section{References}

[1] J. Zachary, ASME Turbo Expo, 3, 607-619 (2012)

[2] H. Brodin, O. Andersson and S. Johansson, ASME Turbo Expo, 5A, GT2013-95878 (2013)

[3] R. Konečná, G. Nicoletto, L. Kunz and A. Bača, Proc. Struct. Integr., 2, 2381-2388 (2016)

[4] J. Suryawanshi, K. G. Prashanth and U. Ramamurty, J. Mater. Sci. Eng. A, 696, 113-121 (2017)

[5] F. Wang, Int. J. Adv. Manuf. Tecnol., 58, 545-551 (2012)

[6] A. Yadollahi, N. Shamsaei, S. M. Thompson, A. Elwany and L. Bian, Int. J. Fatigue, 94, 218-235 (2017)

[7] D. Agius, K. I. Kourousis, C. Wallbrink and T. Song, J. Mater. Sci. Eng. A, 701, 85-100 (2017)

[8] O. Andersson, A. Graichen, H. Brodin and V. Navrotsky, J. Eng. Gas Turbine Power, 139, 031506 (2016)

[9] N. S. Ottosen and M. Ristinmaa, The Mechanics of Constitutive Modeling (2005)

[10] R. Hill, Proc. R. Soc. A, 193, 281-297 (1948)

[11] N. Ohno and J.-D. Wang, Int. J. Plast., 9, 375-390 (1993)

[12] K. N. Smith, T. Topper and P. Watson, J. Mater., 5, 767-778 (1970)

[13] ABAQUS, 6.12 Documentation, (2014).

[14] P. Kanagarajah, F. Brenne, T. Niendorf and H. J. Maier, J. Mater. Sci. Eng. A, 588, 188-195 (2013) 